\title{
Effective feedback for maintenance of competence: from data delivery to trusting dialogues
}

\author{
Kevin W. Eva PhD, Glenn Regehr PhD
}

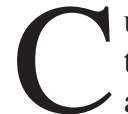
urrent models of maintenance of competence and self-regulation within medicine are largely formalizations of the instruction "Physician, know thyself." For the individual, appreciating the limits of one's abilities is considered a core aspect of professionalism. Failure in this regard threatens quality of care. For the medical profession, self-direction has been established as a foundation on which maintenance of competence (and maintenance of certification programs) are built. Failure in this regard threatens the privilege of self-regulation granted to the profession. In this commentary, we use a cognitive perspective to outline the insufficiency of reliance on self-assessment and the inadequacy of simple efforts to redress its limitations. Our goals are to raise awareness of the complexity of the issue and offer some practical solutions.

Unquestionably, we each have more information on which to judge our own abilities than do external observers. However, this very wealth of information may prevent us from generating accurate impressions of our abilities and from properly appreciating the value of feedback from other sources. ${ }^{1}$ An extensive body of research has shown the robust inaccuracy of self-assessment ${ }^{2}$ and revealed several cognitive mechanisms by which we fool ourselves into believing that we have privileged insight into our own capacities, thereby undermining the benefit we might gain in taking guidance from others. ${ }^{3}$ This literature raises fundamental concerns about the health profession's conception of self-assessment as the pathway to improved performance.

For many decades, the response to these findings has been the use of targeted training to improve practitioners' self-assessment abilities. ${ }^{4}$ Given that it is easy to identify lack of insight in others and difficult to appreciate the same flaws in ourselves, most of us tend to believe that we are above average at self-assessment. ${ }^{5}$ Thus, it is perhaps not surprising that educational efforts have commonly, albeit mistakenly, been directed toward training "them" to be as good at selfassessment as are "we."

As the ubiquity and persistence of poor selfassessment has become more fully appreciated, proposed solutions have begun shifting toward establishing computer systems that will enable physicians to access "better data" on their practice population and treatment habits. ${ }^{6}$ Although such data are important, emphasis on "getting the data right" may perpetuate the problem of self-assessment rather than solve it. That is, although this new approach has shifted emphasis from "improving the process of self-assessment" to "improving the data on which self-assessments are based," it still relies on the individual's ability to incorporate these data into an "accurate" sense of self. Thus, although this enterprise is often described as providing better feedback, its almost exclusive focus on improving the data likely pays too little attention to the conceptualization of meaningful feedback as an iterative, interactive process.

Feedback has been a topic of research and theorizing in the educational and psychology literatures for over a century. ${ }^{1}$ However, the dominant emphasis of this work has been on mechanisms of effective delivery with relatively little focus on the person who receives the feedback, the relationship between recipient and deliverer, or the context in which the feedback is delivered. ${ }^{7}$ One fundamental error in this regard has been to treat feedback recipients as rational beings. Health professionals in particular are generally well-meaning and intelligent individuals who have dedicated their lives to the altruistic good. It is assumed, therefore, that if we provide

\section{KEY POINTS}

- Effective models of maintenance of competence are a fundamental determinant of both the quality of care provided by individual health care professionals and the self-regulating privilege of health care professions

- People perceive data selectively and, as a result, self-perceptions are not trustworthy indicators of ability despite the volume of experiences through which they are generated.

- People are unlikely to change in response to data-delivery methods that attack their self-perceptions.

- Dialogue between peers or with a coach in a trusting relationship that aims at building on a physician's strengths is more likely to be an effective mechanism for practice change and, therefore, maintenance of competence. 
them with compelling and meaningful data about the limits of their practice, they will turn their good intentions into improved performance and, therefore, better health care. Research on cognitive dissonance, however, suggests otherwise. ${ }^{8}$

Cognitive dissonance is the discomfort created by trying to maintain 2 conflicting beliefs at the same time. We are motivated to lessen such discomfort and, in response, tend to alter one of the beliefs. Accepting and incorporating corrective feedback, even in the form of "objective" practice data, requires the acknowledgement that one is performing suboptimally. Such an understanding is, by definition, in conflict with the belief that one is serving one's patients well. Because it is easier to question the data than to question oneself, this pair of conflicting beliefs will often be resolved by discounting the feedback rather than altering one's sense of self as a competent clinician. ${ }^{9}$ In fact, the literature on self-efficacy suggests that this discounting may have value. Having a belief that one can accomplish a goal increases the likelihood of accomplishing it. ${ }^{10}$ Thus, the drive to sustain a positive self-concept might be important to good performance, and threats to positive self-concept should be defended against.

These findings have implications for constructions of feedback and its role in practice improvement. They suggest that providing clear and accurate data are not sufficient. Rather, having a coach or peer group who can help the recipient grapple with available data while clearly keeping the best interests and well-being of the recipient at heart is likely vital. In these coaching interactions, effective guidance should not focus on helping people understand that they are weak by pointing out their flaws. Rather, it should focus on negotiating how their practices could evolve to achieve meaningful goals and what might need to be learned to achieve these goals. In fact, the findings described here suggest that feedback should not be about the delivery or receipt of information at all. Instead, it should be about identifying appropriate challenges (i.e., desirable difficulties) that enable individuals to maintain their perspective as competent and conscientious practitioners while also continually evolving their practice. ${ }^{1}$

In recent years, there has been growing awareness of the value of physician-patient interaction that is based on a foundation of good communication, preservation of the dignity and integrity of the patient, and collaborative decision-making. We feel that similar principles need to be translated into physician-physician and educatortrainee dynamics in a manner that takes into account the motivations, desires and perspectives of feedback recipients, thus enabling them to improve themselves regardless of their ability to know themselves.

\section{References}

1. Eva KW, Regehr G, Gruppen LD. Blinded by "insight": selfassessment and its role in performance improvement. In: Hodges $\mathrm{BD}$, Lingard L, editors. The question of competence: reconsidering medical education in the twenty-first century. New York (NY): Cornell University Press; 2012. p. 131-54.

2. Davis DA, Mazmanian PE, Fordis M, et al. Accuracy of physician self-assessment compared with observed measures of competence: a systematic review. JAMA 2006;296:1094-102.

3. Dunning D, Heath C, Suls J. Flawed self-assessment: implications for health, education, and the workplace. Psychol Sci Public Interest 2004;5:69-106

4. Gordon MJ. Self-assessment programs and their implications for health professions training. Acad Med 1992;67:672-9.

5. Pronin E, Lin DY, Ross L. The bias blind spot: perceptions of bias in self versus others. Pers Soc Psychol Bull 2002;28:369-81.

6. Galbraith RM, Hawkins RE, Holmboe ES. Making self-assessment more effective. J Contin Educ Health Prof 2008;28:20-4.

7. Boud D, Molloy E. Rethinking models of feedback for learning. the challenge of design. Assess Eval High Educ 2012; June 1 [Epub ahead of print]

8. Tavris C, Aronson E. Mistakes were made (but not by me): why we justify foolish beliefs, bad decisions, and hurtful acts. New York (NY): Harcourt; 2007. p. 4.

9. Eva KW, Armson H, Holmboe E, et al. Factors influencing responsiveness to feedback: on the interplay between fear, confidence, and reasoning processes. Adv Health Sci Educ Theory Pract 2012;17:15-26.

10. Eva KW, Regehr G. Self-assessment in the health professions: a reformulation and research agenda. Acad Med 2005;80(Suppl 10): S46-54.

Affiliations: Kevin Eva and Glenn Regehr are with the Centre for Health Education Scholarship, University of British Columbia. Kevin Eva is with the Department of Medicine and Glenn Regehr is with the Department of Surgery, University of British Columbia, Vancouver, BC.

Contributors: Both authors contributed to the ideas outlined in this article. Kevin Eva drafted the manuscript. Both authors critically revised the manuscript and approved the final version submitted for publication. 